\title{
Normal cones to infinite intersections
}

\author{
Thomas I. Seidman*
}

December 13, 2009

\begin{abstract}
For sets given as finite intersections $\mathcal{A}=\bigcap_{k=1}^{K} \mathcal{A}_{k}$ the basic normal cone $N(\bar{x} ; \mathcal{A})$ is given as $\sum_{k} N\left(\bar{x} ; \mathcal{A}_{k}\right)$, but such a result is not, in general, available for infinite intersections. A comparable characterization of $N(\bar{x} ; \mathcal{A})$ is obtained here for a class of such infinite intersections.
\end{abstract}

Key words: Nonsmooth analysis, normal cone, intersection rule.

AMS subject classifications.

\section{Introduction}

A fundamental concern in variational analysis is the characterization through first-order optimality conditions of the solution of a constrained minimization problem

$$
\text { Minimize: } f(x) \quad \text { subject to: } x \in \mathcal{A} \text {. }
$$

Under quite general conditions the first-order conditions on a local minimizer $\bar{x}$ are known [4, Prop. 5.1] to take the form

$$
-[\nabla f](\bar{x}) \in \hat{N}_{0}(\bar{x} ; \mathcal{A}) \subset N(\bar{x} ; \mathcal{A})
$$

where $N(\bar{x} ; \mathcal{A})$ denotes the basic normal cone at $\bar{x}$ (e.g., in the sense of Mordukhovich [3]; see Definitions 2.1, 2.2) to the admissible set $\mathcal{A}$. Thus we are led to the task of computing $N(\bar{x} ; \mathcal{A})$ from the specification provided for $\mathcal{A}$.

* Department of Mathematics and Statistics, University of Maryland Baltimore County, Baltimore, MD 21250, USA (seidman@math.umbc.edu). 
Constraints in large-scale problems are often generated by replicating a limited number of constraint prototypes over large index sets. In particular, we may consider, as prototype, constraints of the form $\varphi \geq 0$ for a scalar function $\varphi$, noting that the constraints in problem (1) are often given in the form of a family of such inequalities

$$
\text { Minimize: } f(x) \quad \text { subject to: } \varphi(x) \geq 0 \text { for } \varphi \in \Phi
$$

where $\Phi$ is a set of constraint functions $\varphi: \mathcal{X} \rightarrow \mathbb{R}$. [While this set of inequalities could be subsumed by a single inequality: $\varphi_{*}(x) \geq 0$ on taking $\varphi_{*}(x)=\inf \{\varphi(x): \varphi \in \Phi\}$, this does not seem helpful. Indeed, we would have $-\operatorname{epi} \varphi_{*}=\bigcap_{\varphi \in \Phi}\{-\operatorname{epi}(\varphi)\}$ so the present consideration of infinite intersections seems as likely to help with differentiation of sup or inf as vice versa. We also note that (3) is frequently seen with the inequality reversed, simply corresponding to the replacement $\varphi \longleftrightarrow-\varphi$.]

Note that (3) means that the admissible set $\mathcal{A}$ is presented as an intersection

$$
\mathcal{A}=\bigcap_{\varphi \in \Phi} \mathcal{A}^{\varphi} \quad \text { with } \mathcal{A}^{\varphi}=\{x: \varphi(x) \geq 0\}=\varphi^{-1}([0, \infty))
$$

and we would like to determine $N(\bar{x} ; \mathcal{A})$ in terms of the functions $\varphi \in \Phi$. When this is a finite intersection $\left(\Phi=\left\{\varphi_{1}, \ldots, \varphi_{K}\right\}\right)$, one has, under quite mild conditions, an 'intersection rule'

$$
N\left(\bar{x} ; \bigcap_{\varphi \in \Phi} \mathcal{A}^{\varphi}\right)=\sum_{\varphi \in \Phi} N\left(\bar{x} ; \mathcal{A}^{\varphi}\right) .
$$

as a special case of [3, Cor. 3.5]. In general, however, no such results are available for infinite intersections. [We do note that (5) is somewhat analogous to differentiating a sum so working with an infinite intersection might be compared to term-by-term differentiation of a series: one expects this to be possible, but under more restrictive hypotheses and perhaps with a modified statement. Certainly we would expect this shift to involve some new ideas - e.g., there is an interchange of limits in the background so we might expect to require some uniformity condition. Even under hypotheses ensuring its validity for each finite subset of $\Phi$, it is clear that the formula (5) will generally be false, as stated, for infinite $\Phi$.] 
Example 1.1. We might consider (4) in a restricted but typical setting: for example $\mathcal{A}$ might be the set of non-negative functions in, e.g., $\mathcal{X}=C(\Omega)$. This is, indeed, of the form (4) we are considering, here with $\Phi$ the set of evaluation functionals $\Phi=\{[x(\cdot) \mapsto x(s)]: s \in \Omega\}$, noting that this is rather special in that $\mathcal{A}$ is here a closed convex cone in $\mathcal{X}$.

Somewhat more generally, we might consider an arbitrary closed convex set in a Banach space $\mathcal{X}$ and note that this is describable as the intersection of all the half-spaces containing it:

$$
\mathcal{A}=\bigcap_{\alpha \in \mathcal{I}} \mathcal{A}_{\alpha} \quad \mathcal{A}_{\alpha}=\left\{x:\left\langle\xi_{\alpha}, x\right\rangle \geq \rho_{\alpha}\right\}
$$

which is of the form (4) with $\varphi \in \Phi$ of the form $\varphi(x)=\langle\xi, x\rangle-\rho$. Given $\bar{x}$ at the boundary of $\mathcal{A}$, the active set of constraints is given by $\Phi_{*}=\{\varphi \in \Phi$ : $\langle\xi, \bar{x}\rangle=\rho\}$ so the set of support functionals at $\bar{x}$ is $\left\{-\xi: \varphi \in \Phi_{*}\right\}$. Since $N\left(\bar{x} ; \mathcal{A}_{\alpha}\right)=\{0\}$ for the inactive constraints $\alpha \in \mathcal{I} \backslash \mathcal{I}_{*}$, we have

$$
N(\bar{x} ; \mathcal{A})=\overline{\operatorname{co}}\left\{N\left(\bar{x} ; \mathcal{A}_{\alpha}\right): \alpha \in \mathcal{I}\right\}
$$

where " $\overline{\mathrm{co}} \mathcal{S}$ " denotes the conical hull of $\mathcal{S}$, i.e., the closure of the convex hull of $\{a \xi: a>0, \xi \in \mathcal{S}\}$, so (7) is the natural interpretation of (5). This characterization depends on our having used the complete set of support functionals in specifying $\mathcal{A}$.

Example 1.2. As another simple example, take $\mathcal{X}=\mathbb{R}, \bar{x}=0$, and let $\varphi_{k}(x)=x+1 / k$. This gives $0=\bar{x}$ in the interior of each $\mathcal{A}^{\varphi_{k}}=[-1 / k, \infty)$ so no given constraint would be active. Here, each $N\left(0 ; \mathcal{A}^{\varphi_{k}}\right)=\{0\}$, while $\mathcal{A}=[0, \infty)$ so $N(0 ; \mathcal{A})=(-\infty, 0] \neq\{0\}$.

Example 1.3. A slightly different example takes $\mathcal{A}$ to be the unit disk centered at the origin of $\mathbb{R}^{2}$ which we present as the infinite intersection of the countable set of half-spaces $\mathcal{A}^{\varphi}$ given by

$$
\varphi_{ \pm k}(x)=1-\left(r_{k}, \pm \sqrt{1-r_{k}^{2}}\right) \cdot x
$$

where $\left(r_{k}\right)$ is an enumeration of the rationals in $[-1,1]$. If we then take $\bar{x}=$ $(1 / \sqrt{2}, 1 / \sqrt{2})$, none of these constraints are active since $\bar{x}$ is in the interior of each of the presenting half-spaces $\mathcal{A}^{\varphi_{k}}$ above and the support functionals $(a, a)$ exactly at $\bar{x}$ do not appear in the specifying $\left\{\varphi_{k}\right\}$. Nevertheless, the 
normal cone $N(\bar{x} ; \mathcal{A})=\{(-a,-a): a \geq 0\}$ is expressible in terms of these through the neighboring support functionals $\left(\varphi_{k}(\bar{x}) \approx 0\right)$ :

$$
N(\bar{x} ; \mathcal{A})=-\bigcap_{\omega>0} \overline{\operatorname{co}}\left\{\varphi_{k}^{\prime}(\bar{x}): \varphi_{k}(\bar{x})<\omega\right\}
$$

noting that $\varphi_{k}(\bar{x}) \leq \omega$ gives a $\varphi_{k}^{\prime}$ in a wedge centered at $(a, a)$ of angular width diminishing to 0 as $\omega \rightarrow 0$.

Example 1.4. Again with $\mathcal{X}=\mathbb{R}^{2}, \bar{x}=(0,0)$, one might consider a quite different variant taking $\varphi_{k}(x, y)=\left\{x\right.$ if $y \geq 0 ; x-k y^{2}$ if $\left.y \leq 0\right\}$. Here each constraint is active at $\bar{x}$ and each $N\left(\bar{x} ; \mathcal{A}^{\varphi_{k}}\right)=\{(-r, 0): r \geq 0\}$. However, $\mathcal{A}=\{(x, y): x, y \geq 0\}$ with $N(\bar{x} ; \mathcal{A})=\{(r, s): r, s \leq 0\}$ and this is not contained in the hull of $\left\{N\left(\bar{x} ; \mathcal{A}^{\varphi_{k}}\right): k=1,2, \ldots\right\}$.

It is clear from Example 1.3 that the active constraints taken from a set of specifying constraints need not be sufficient and we must also consider 'almost active' constraints, for which $\varphi(\bar{x}) /\|\xi\|$ is small although not exactly 0 . We thus have a simultaneous concern for two questions:

1. The defining functionals are nonlinear, although moderately smooth, so the set $\mathcal{A}$ need not be convex - and, of course, there may be infinitely many such defining constraint functionals.

2. We are concerned that the set of 'presenting functionals' defining $\mathcal{A}$ may not be complete: the active constraints (support functionals at $\bar{x}$ ) might not appear at all.

Our goal here is to obtain a more general (nonconvex) version of the convex case (7), replacing the affine functionals $\psi=\psi^{\rho, \xi}$ appearing there by an infinite set $\Phi$ of nonlinear Fréchet differentiable functionals $\varphi: \mathcal{X} \rightarrow \mathbb{R}$ giving (4) while taking account of 'almost active' constraints in formulating the result. [We also note in this a need for some uniformity condition to rule out consideration of Example 1.4.]

Remark 1.5. We are motivated here by considerations [2] involving a hyperelastic inextensible rod with centerline $x(\cdot) \in \mathcal{X}=H^{2}\left([0,1] \rightarrow \mathbb{R}^{3}\right)$. The relevant constraint there is that this rod, viewed as a solid tube of radius $\rho$ around the centerline, should not intersect itself. Geometrically, this means that while local pairs (close together along the rod) are necessarily physically 
close, for nonlocal pairs - $\mathcal{P}=\left\{\left(s, s^{\prime}\right) \in[0,1]^{2}:\left|s-s^{\prime}\right| \geq \pi \rho\right\}$ — we must have at least a $2 \rho$ separation. Thus, we are asking that $\varphi(\bar{x}) \geq 0$ for each $\varphi$ of the form

$$
\varphi(x)=\left|x(s)-x\left(s^{\prime}\right)\right|-2 \rho \quad \text { with }\left(s, s^{\prime}\right) \in \mathcal{P} \text { as above. }
$$

This constraint can clearly be formulated as requiring that $\bar{x}$ should lie in the (uncountably infinite) intersection of the sets $\mathcal{A}^{\varphi}$ with $\varphi$ ranging over $\Phi$ : the functions of (9), indexed by the set $\mathcal{P}$ of nonlocal pairs. We easily see that this is not a convex constraint - one easily constructs pairs of configurations not involving self-intersection such that the average does intersect itself so we cannot expect convex analysis to be adequate for the treatment of this problem.

In this setting we note that active constraints correspond to contact pairs: $\mathcal{P}_{*}=\left\{\left(s, s^{\prime}\right) \in \mathcal{P}:\left|\bar{x}(s)-\bar{x}\left(s^{\prime}\right)\right|=2 \rho\right\}$. For these we would have, by the chain rule, $N\left(\bar{x} ; \mathcal{A}^{\varphi}\right)=\left\{-r \xi^{\varphi}: r \geq 0\right\}$ where $\xi^{\varphi}=\varphi^{\prime}(\bar{x})$. We would then expect the normal cone $N(\bar{x} ; \mathcal{A})$ to be expressible in terms of these while being aware that this intuition is necessarily somewhat suspect in view of Example 1.2.

Next, we note that the normal cone is constructed in $\mathcal{X}^{*}$ which here is topologized as $H^{-2}\left([0,1] \rightarrow \mathbb{R}^{3}\right)$. On the other hand, while the functions $\varphi$ of (9) are initially given on $\mathcal{X}$, they may certainly be extended as functions on the larger space $\mathcal{Y}=C\left([0,1] \rightarrow \mathbb{R}^{3}\right)$ and are also Fréchet differentiable when considered there. We would hope, then, that $N(\bar{x} ; \mathcal{A})$ would then turn out to be in $\mathcal{Y}^{*}$ so its elements would be vector-valued measures with support in the set of contact points: $s \in[0,1]$ for which there is some $s^{\prime}$ with $\left(s, s^{\prime}\right) \in \mathcal{P}_{*}$.

From this point of view, the culmination of this paper is Theorem 4.3 below, which will provide these desired results, subject to the contextual verification in [2] of the abstract hypotheses imposed here.

\section{Setting, notation, hypotheses}

We recall from [3] Mordukhovich's definition of $N(\bar{z} ; \Omega) \subset \mathcal{Z}^{*}$, the basic normal cone at a point $\bar{z} \in \mathcal{Z}$ to a subset $\Omega$ of a Banach space $\mathcal{Z}$.

Definition 2.1. For $\hat{z} \in \Omega \subset \mathcal{Z}$ and $\varepsilon \geq 0$, we have $\zeta \in \hat{N}_{\varepsilon}(\hat{z} ; \Omega) \subset \mathcal{Z}^{*}$ if: $\lim \sup \left\langle\zeta, z_{k}\right\rangle \leq \varepsilon$ for every sequence $\left(t_{k}, z_{k}\right)$ in $\mathbb{R}_{+} \times \mathcal{Z}$ such that: $\left\|z_{k}\right\|_{\mathcal{Z}}=1$ and $t_{k} \rightarrow 0$ in $\mathbb{R}_{+}$with $\left(\hat{z}+t_{k} z_{k}\right) \in \Omega$. 
Definition 2.2. $\bar{\zeta}$ is in the basic normal cone $N(\bar{z} ; \Omega) \subset \mathcal{Z}^{*}$ if:

there is a sequence $\left(\varepsilon_{j}, \hat{z}_{j}, \zeta_{j}\right)$ in $\mathbb{R} \times \mathcal{Z} \times \mathcal{Z}^{*}$ such that: $\varepsilon_{j} \downarrow 0, \hat{z}_{j} \rightarrow \bar{z}$, $\zeta_{j}{ }^{*} \bar{\zeta}\left(\right.$ weak- ${ }^{*}$ convergence in $\left.\mathcal{Z}^{*}\right)$ and $\zeta_{j} \in \hat{N}_{\varepsilon_{j}}\left(\hat{z}_{j} ; \Omega\right)$.

[Note that only a neighborhood $\mathcal{U}$ of $\bar{z}$ is relevant for this.]

As the initial setting for our considerations we begin with a Banach space $\mathcal{X}$, a point $\bar{x} \in \mathcal{X}$, a neighborhood $\bar{x} \in \mathcal{U} \subset \mathcal{X}$. We then have a family $\Phi$ of scalar functions $\varphi: \mathcal{U} \rightarrow \mathbb{R}$ and wish to consider the set $\mathcal{A}$ on which each $\varphi \in \Phi$ is non-negative, i.e.,

$$
\mathcal{A}=\{x \in \mathcal{U}: \varphi(x) \geq 0 \text { for all } \varphi \in \Phi\}
$$

Note that $\mathcal{A}$ is the infinite intersection of singly constrained sets:

$$
\mathcal{A}=\bigcap_{\varphi \in \Phi} \mathcal{A}^{\varphi} \quad \mathcal{A}^{\varphi}=\{x \in \mathcal{U}: \varphi(x) \geq 0\}
$$

Our first hypothesis here is that:

Each $\varphi \in \Phi$ is Fréchet differentiable on $\mathcal{U} \subset \mathcal{X}$ with $0 \neq \varphi^{\prime}(\bar{x}) \in \mathcal{X}^{*}$.

For each $\varphi \in \Phi$ it is now convenient to define

$$
\omega^{\varphi}=\varphi(\bar{x}), \quad \xi^{\varphi}=\varphi^{\prime}(\bar{x}) .
$$

Since each set $\mathcal{A}^{\varphi}$ is independent of any scaling of $\varphi$, we will assume, with no loss of generality, the normalization

$$
\left\|\xi^{\varphi}\right\|_{\mathcal{X}^{*}}=1 \quad \text { for each } \varphi \in \Phi
$$

As $\bar{\omega} \rightarrow 0$, the sets

$$
\Phi^{\bar{\omega}}=\left\{\varphi \in \Phi: \omega^{\varphi} \leq \bar{\omega}\right\}
$$

then give the 'almost active' constraints in $\Phi$. [Only $\varphi \in \Phi^{0}$ are actually active constraints at $\bar{x}$, but it is easily possible to have $\Phi^{0}$ empty yet still have $\mathcal{A}$ constrained at $\bar{x}$ 'in the limit' so we cannot simply discard all the individually inactive constraints. It is important to note that the normalization (13) will affect the specification of $\Phi^{\omega}$ for $\omega>0$ although it does not affect $\mathcal{A}$. ] 
We next impose a uniformity condition on $\Phi$, assuming that the derivative functions $\varphi^{\prime}: \mathcal{X} \supset \mathcal{U} \rightarrow \mathcal{X}^{*}$ are equicontinuous at $\bar{x}-$ i.e.,

For each $\gamma>0$ there exists $\beta=\beta(\gamma)>0$ such that:

for all $x \in \mathcal{U}$ with $\|x-\bar{x}\|_{\mathcal{X}} \leq \beta(\gamma)$ one has

$$
\left\|\varphi^{\prime}(x)-\varphi^{\prime}(\bar{x})\right\|_{\mathcal{X}^{*}} \leq \gamma \text { for all } \varphi \in \Phi .
$$

Our final hypothesis here is a qualification condition: for some $\omega>0$, one has

$$
\begin{aligned}
& \text { There exist } x_{*} \text { with }\left\|x_{*}\right\|=1 \text { and } \sigma>0 \text { such that, } \\
& \text { if }\|x\|_{\mathcal{X}} \leq \sigma \text { and } \varphi \in \Phi^{\omega} \text { then }\left\langle\xi^{\varphi}, x_{*}+x\right\rangle \geq 0 \text {. }
\end{aligned}
$$

This just means that the set

$$
\mathcal{B}^{\omega}=\left\{x \in \mathcal{X}:\left\langle\xi^{\varphi}, x\right\rangle \geq 0 \text { for each } \varphi \in \Phi^{\omega}\right\},
$$

has nonempty interior, specifically that it contains the ball centered at $x_{*}$ with radius $\sigma$. Clearly, (16) for some $\omega>0$ implies (16) for all $0<\omega^{\prime}<\omega$. We also note that the inequality $\left\langle\xi^{\varphi}, x_{*}\right\rangle \geq\left\langle\xi^{\varphi}, x\right\rangle$ for all $x$ with $\|x\|_{\mathcal{X}} \leq \sigma$ gives

$$
\left\langle\xi^{\varphi}, x_{*}\right\rangle \geq \sigma\left\|\xi^{\varphi}\right\|_{\mathcal{X}^{*}} \quad \text { for each } \varphi \in \Phi^{\omega} .
$$

\section{Main theorem}

Theorem 3.1. Assume the hypotheses (11), (15), (16). Then the basic normal cone at $\bar{x}$ to the infinite intersection $\mathcal{A}=\bigcap\left\{\mathcal{A}^{\varphi}: \varphi \in \Phi\right\}$ satisfies

$$
N(\bar{x} ; \mathcal{A}) \subset \bigcap_{\omega>0} N\left(0 ; \mathcal{B}^{\omega}\right)
$$

with $\mathcal{B}^{\omega}$ given by (17), using (14).

Proof: We begin by setting

$$
\delta(\varepsilon)=\delta(\varepsilon, \omega, M)=\frac{1}{2} \min \left\{\frac{\omega}{2}, \beta(1), \beta\left(\frac{3 \varepsilon \sigma}{8 M}\right)\right\}
$$

where we obtain $\beta(\cdot)$ from (15) with $\gamma=1$ and with $\gamma=3 \varepsilon \sigma / 8 M$, taking $\sigma$ from (16) with $\left\|x_{*}\right\|_{\mathcal{X}}=1$. 
Our first step is then to show the key inclusion:

$$
\begin{aligned}
\text { If }\|\hat{x}-\bar{x}\|_{\mathcal{X}} & \leq \delta(\varepsilon), \text { then } \\
\{\xi & \left.\in \hat{N}_{\varepsilon}(\hat{x} ; \mathcal{A}):\|\xi\|_{\mathcal{X}^{*}} \leq M\right\} \subset \hat{N}_{3 \varepsilon}\left(0 ; \mathcal{B}^{\omega}\right) .
\end{aligned}
$$

Suppose (21), using (20), were false. Then we would have $\|\hat{x}-\bar{x}\|_{\mathcal{X}} \leq \delta(\varepsilon)$ and $\xi \in \hat{N}_{\varepsilon}(\hat{x} ; \mathcal{A})$ with $\|\xi\|_{\mathcal{X}^{*}} \leq M$, yet $\xi \notin \hat{N}_{3 \varepsilon}\left(0 ; \mathcal{B}^{\omega}\right)$. I.e., since $\mathcal{B}^{\omega}$ is a cone, Definition 2.1 just says that there is some $\tilde{x} \in \mathcal{B}^{\omega}$ with $\|\tilde{x}\|_{\mathcal{X}}=1$ and $\langle\xi, \tilde{x}\rangle>3 \varepsilon$. [We note that, with $\|\tilde{x}\|_{\mathcal{X}}=1$, this requires $3 \varepsilon \leq\|\xi\|_{\mathcal{X}^{*}}$.]

We wish to use this $\tilde{x}$ to show, in applying Definition 2.1, that we could not, in fact, have $\xi \in \hat{N}_{\varepsilon}(\hat{x} ; \mathcal{A})$; we will set

$$
v=\tilde{x}+\frac{\varepsilon}{\|\xi\|_{\mathcal{X}^{*}}} x_{*}
$$

noting that $\|v\|_{\mathcal{X}} \leq 4 / 3$. We then have

$$
\begin{aligned}
\langle\xi, v\rangle & =\langle\xi, \tilde{x}\rangle+\frac{\varepsilon}{\|\xi\|_{\mathcal{X}^{*}}}\left\langle\xi, x_{*}\right\rangle \\
& \geq 3 \varepsilon-\frac{\varepsilon}{\|\xi\|_{\mathcal{X}^{*}}}\|\xi\|_{\mathcal{X}^{*}}=2 \varepsilon \geq \frac{3}{2} \varepsilon\|v\|_{\mathcal{X}} .
\end{aligned}
$$

so the condition $\lim \sup \left\langle\xi, z_{k}\right\rangle \leq \varepsilon$ of Definition 2.1 would fail for the normalized sequence $z_{k}=v /\|v\|_{\mathcal{X}}$. This last ensures that $\xi \notin \hat{N}_{\varepsilon}(\hat{x} ; \mathcal{A})$ if we can show that $\hat{x}+t_{k} z_{k} \in \mathcal{A}$, i.e., that

$$
\varphi(\hat{x}+t v) \geq 0 \text { for each } \varphi \in \Phi
$$

for small enough $t>0$, considering $t=t_{k} /\|v\|_{\mathcal{X}}$.

We will split the task of verifying (24) into two parts: for $\varphi \in \Phi \backslash \Phi^{\omega}$ and for $\varphi \in \Phi^{\omega}$. In each case we will use the assumption that $\|\hat{x}-\bar{x}\|_{\mathcal{X}} \leq \delta-$ whence, (also with $t<\delta /\|v\|_{\mathcal{X}}$ ) one has $\left\|x_{\tau}-\bar{x}\right\|_{\mathcal{X}}<2 \delta$ for $0 \leq \tau \leq 1$ with either

$$
x_{\tau}=\tau(\hat{x}+t v)+(1-\tau) \hat{x} \quad \text { or } \quad x_{\tau}=\tau(\hat{x}+t v)+(1-\tau) \bar{x}
$$

giving $x_{\tau}$ on the segment joining $(\hat{x}+t v)$ either to $\hat{x}$ or to $\bar{x}$. Thus we will be able to apply the hypothesis (15) with (20) to estimate $\left\|\varphi^{\prime}\left(x_{\tau}\right)-\xi^{\varphi}\right\|_{\mathcal{X}^{*}} \leq \gamma$, as appropriate. 
For the first case we have $\varphi(\bar{x})=\omega^{\varphi}>\omega$ and estimate $\varphi(\hat{x}+t v)$ by

$$
\begin{aligned}
\varphi(\hat{x}+t v)-\varphi(\bar{x}) & =\int_{0}^{1}\left\langle\varphi^{\prime}\left(x_{\tau}\right), \hat{x}+t v-\bar{x}\right\rangle d \tau \\
& =\left\langle\xi^{\varphi}+R, \hat{x}-\bar{x}+t v\right\rangle \\
& \geq-\left(1+\|R\|_{\mathcal{X}^{*}}\right)(2 \delta)
\end{aligned}
$$

with

$$
R=\int_{0}^{1}\left[\varphi^{\prime}\left(x_{\tau}\right)-\varphi^{\prime}(\bar{x})\right] d \tau \quad \text { so }\|R\|_{\mathcal{X}^{*}} \leq \gamma=1
$$

since $2 \delta \leq \beta(1)$. Our choice of $\delta$ also gives $2 \delta<\omega / 2$ so

$$
\varphi(\hat{x}+t v)=\varphi(\bar{x})+\left\langle\xi^{\varphi}+R, \hat{x}-\bar{x}+t v\right\rangle>\omega-4 \delta \geq 0 .
$$

For the second case we estimate $\varphi(\hat{x}+t v)$, much as above, by

$$
\begin{aligned}
\varphi(\hat{x}+t v)-\varphi(\hat{x}) & =t \int_{0}^{1}\left\langle\varphi^{\prime}\left(x_{\tau}\right), v\right\rangle d \tau \\
& =t\left[\left\langle\xi^{\varphi}, \tilde{x}\right\rangle+\left(\varepsilon /\|\xi\|_{\mathcal{X}^{*}}\right)\left\langle\xi^{\varphi}, x_{*}\right\rangle+\langle R, v\rangle\right]
\end{aligned}
$$

now with

$$
R=\int_{0}^{1}\left[\varphi^{\prime}\left(x_{\tau}\right)-\varphi^{\prime}(\hat{x})\right] d \tau \quad \text { so }\|R\|_{\mathcal{X}^{*}} \leq 2 \gamma=\frac{3 \varepsilon \sigma}{4 M}
$$

since $\left|\varphi^{\prime}\left(x_{\tau}\right)-\varphi^{\prime}(\hat{x})\right| \leq\left|\varphi^{\prime}\left(x_{\tau}\right)-\varphi^{\prime}(\bar{x})\right|+\left|\varphi^{\prime}(\hat{x})-\varphi^{\prime}(\bar{x})\right|$ and $2 \delta \leq \beta(3 \varepsilon \sigma / 8 M)$. We note that $\varphi(\hat{x}) \geq 0$ since we have $\hat{x} \in \mathcal{A}$ and that $\left\langle\xi^{\varphi}, \tilde{x}\right\rangle \geq 0$ since $\varphi \in \Phi^{\omega}$ and $\tilde{x} \in \mathcal{B}^{\omega}$; we also can use (18). Thus (25) gives

$$
\begin{aligned}
\varphi(\hat{x}+t v) & \geq t\left[\left(\varepsilon /\|\xi\|_{\mathcal{X}^{*}}\right)\left\langle\xi^{\varphi}, x_{*}\right\rangle+\langle R, v\rangle\right] \\
& \geq t\left[\left(\varepsilon /\|\xi\|_{\mathcal{X}^{*}}\right) \sigma-\|R\|_{\mathcal{X}^{*}}\|v\|_{\mathcal{X}}\right] \\
& \geq t[(\varepsilon / M) \sigma-(3 \varepsilon \sigma / 4 M)(4 / 3)]=0 .
\end{aligned}
$$

This completes the proof of the key inclusion (21) and we now return to (19) for our conclusion.

Suppose, then, we have $\bar{\xi} \in N(\bar{x}, ; \mathcal{A})$ so, by Definition 2.2 , there are sequences $\varepsilon_{k} \downarrow 0, \hat{x}_{k} \rightarrow \bar{x}$, and $\xi_{k} \in \hat{N}_{\varepsilon_{k}}\left(\hat{x}_{k} ; \mathcal{A}\right)$ such that $\xi_{k} \stackrel{*}{\rightarrow} \bar{\xi}$ in $\mathcal{X}^{*}$; necessarily $\left(\xi_{k}\right)$ is bounded so, for some fixed $M$, we have $\left\|\xi_{k}\right\|_{\mathcal{X}^{*}} \leq M$. We can now choose $k=k(j)$ recursively so that

$$
\left\|\hat{x}_{k(j)}-\bar{x}\right\| \leq \delta\left(\varepsilon_{j} ; \omega, M\right), \quad k(j)>k(j-1),
$$


and then set $\tilde{\varepsilon}_{j}=\varepsilon_{j}, \tilde{\xi}_{j}=\xi_{k(j)}$. Note that each $k(j) \geq j$ so $\varepsilon_{k(j)} \leq \varepsilon_{j}=\tilde{\varepsilon}_{j}$ whence

$$
\tilde{\xi}_{j}=\xi_{k(j)} \in \hat{N}_{\varepsilon_{k(j)}}\left(\hat{x}_{k(j)} ; \mathcal{A}\right)=\hat{N}_{\varepsilon_{k(j)}}\left(\tilde{x}_{j} ; \mathcal{A}\right) \subset \hat{N}_{\tilde{\varepsilon}_{j}}\left(\tilde{x}_{j} ; \mathcal{A}\right)
$$

since Definition 2.1 gives $\hat{N}_{\varepsilon}(x ; \mathcal{A}) \subset \hat{N}_{\varepsilon^{\prime}}(x ; \mathcal{A})$ when $\varepsilon \leq \varepsilon^{\prime}$. We also have $\left\|\tilde{x}_{j}-\bar{x}\right\| \leq \delta\left(\tilde{\varepsilon}_{j}\right)$ by our construction so (21) applies with $\varepsilon=\tilde{\varepsilon}_{j}$ and $\xi=\tilde{\xi}_{j}$. We thus have $\tilde{\xi}_{j} \in \hat{N}_{3 \tilde{\varepsilon}_{j}}\left(0 ; \mathcal{B}^{\omega}\right)$. Clearly $3 \tilde{\varepsilon}_{j} \downarrow 0$ and $0 \rightarrow 0$ while, as a subsequence of $\xi_{k}$ we have $\tilde{\xi}_{j} \stackrel{*}{\rightarrow} \bar{\xi}$.

Using Definition 2.2 again, this means that each such $\bar{\xi} \in N(\bar{x}, ; \mathcal{A})$ must be in $N\left(0 ; \mathcal{B}^{\omega}\right)$; since this holds for each $\omega>0$, we have (19).

\section{Further remarks}

We would like to clarify the meaning of Theorem 3.1 by providing a more geometric characterization of the right hand side of (19).

Corollary 4.1. Under the hypotheses of the Theorem, with $\mathcal{X}$ reflexive, one has

$$
N(\bar{x} ; \mathcal{A}) \subset-\bigcap_{\omega>0} \overline{\mathrm{co}} \Xi^{\omega} \quad \text { where } \Xi^{\omega}=\left\{\varphi^{\prime}(\bar{x}): \varphi \in \Phi, \omega^{\varphi} \leq \omega\right\} .
$$

which corresponds a bit more closely to (5) and, we note is precisely parallel to (8) in Example 1.3.

ProOF: We note that (17) gives

$$
\mathcal{B}^{\omega}=\left\{x \in \mathcal{X}:\langle\xi, x\rangle \geq 0 \forall \xi \in \Xi^{\omega}\right\}=\left\{x \in \mathcal{X}:\langle\xi, x\rangle \geq 0 \forall \xi \in \overline{\mathrm{co}} \Xi^{\omega}\right\} .
$$

On the other hand, since $\mathcal{B}^{\omega}$ is a convex cone (pointed at 0 ) we have from this (following, e.g., [1, p. 136], [3, Prop. 1.5]) that

$$
N\left(0 ; \mathcal{B}^{\omega}\right)=\hat{N}\left(0 ; \mathcal{B}^{\omega}\right)=\left\{\xi \in \mathcal{X}^{*}:\langle\xi, x\rangle \leq 0 \forall x \in \mathcal{B}^{\omega}\right\} .
$$

Given reflexivity, one now easily sees that $N\left(0 ; \mathcal{B}^{\omega}\right)=-\overline{\mathrm{co}} \Xi^{\omega}$ and the result is then immediate from Theorem 3.1. 
Remark 4.2. Suppose we could strengthen the qualification condition (16) to a similar condition with respect to another, weaker, Banach space $\mathcal{Y}$ i.e., with $\mathcal{X}$ (densely) embedded in $\mathcal{Y}$ so also $\mathcal{Y}^{*} \hookrightarrow \mathcal{X}^{*}$. We would thus be requiring that, for some $\omega>0$,

There exist $y_{*} \in \mathcal{X}$ and $\sigma^{\prime}>0$ such that, for all $\varphi \in \Phi^{\omega}, x \in \mathcal{X}$,

$$
\text { if }\|x\|_{\mathcal{Y}} \leq \sigma^{\prime} \text {, then }\left\langle\xi^{\varphi}, y_{*}+x\right\rangle \geq 0 \text {. }
$$

This just means that the intersection of $\mathcal{X} \subset \mathcal{Y}$ with the ball in $\mathcal{Y}$ centered at $y_{*}$ with radius $r^{\prime}=\sigma^{\prime} /\left\|y_{*}\right\|_{\mathcal{Y}}$ is contained in $\mathcal{B}^{\omega}$. The condition ensures that if $\xi \in \Xi^{\omega} \subset \mathcal{X}^{*}$, then $|\langle\xi, x\rangle| \leq\left\langle\xi, y_{*}\right\rangle$ whenever $\|x\|_{\mathcal{Y}} \leq \sigma$ so $\xi$ must extend by continuity to $\mathcal{Y}$ with $\|\xi\|_{\mathcal{Y}_{*}} \leq\left\langle\xi, y_{*}\right\rangle / \sigma$. In particular, (26) implies that each $\xi^{\varphi}$ is actually in $\mathcal{Y}^{*}$. [We also note that (26) implies (16) with $\sigma=K \sigma^{\prime}$ where $K$ is the norm of the embeddings $\mathcal{X} \hookrightarrow \mathcal{Y}$ and $\mathcal{Y}^{*} \hookrightarrow \mathcal{X}^{*}$.]

As noted, each $\xi^{\varphi}$ will then be in $\mathcal{Y}^{*}$ and we now define

$$
\hat{\mathcal{B}}^{\omega}=\left\{y \in \mathcal{Y}:\left\langle\xi^{\varphi}, y\right\rangle \geq 0 \text { for all } \varphi \in \Phi^{\omega}\right\},
$$

noting that $\hat{\mathcal{B}}^{\omega}$ is just the $\mathcal{Y}$-closure of $\mathcal{B}^{\omega} \hookrightarrow \mathcal{Y}$. Of course, when we compute $N\left(0 ; \hat{\mathcal{B}}^{\omega}\right)$, etc., we are now applying Definition 2.2 with $\mathcal{Z}=\mathcal{Y}$.

Theorem 4.3. Assume the hypotheses (11), (15), (26). Then the basic normal cone at $\bar{x}$ to the infinite intersection $\mathcal{A}=\cap\left\{\mathcal{A}^{\varphi}: \varphi \in \Phi\right\}$ satisfies

$$
N(\bar{x} ; \mathcal{A}) \subset \bigcap_{\omega>0} \hat{N}_{0}\left(0 ; \hat{\mathcal{B}}^{\omega}\right)
$$

where, of course, the right hand side is to be computed in $\mathcal{Y}^{*} \hookrightarrow \mathcal{X}^{*}$.

Proof: Immediate from Theorem 3.1 and the discussion above.

Remark 4.4. If we think of the construction of $\bigcap_{\varphi \in \Phi} \mathcal{A}^{\varphi}$ as a limit of partial intersections, e.g., considering an increasing sequence of (finite) subsets of $\Phi$, then our concerns here seem equivalent to investigating continuous dependence of the normal cone $N(\bar{x} ; \Omega)$ on the set $\Omega$ - at least for monotone (nested) convergence of the argument.

Results of the sort obtained here must certainly also be relevant for 
computational approximation to constrained minimizers where, given appropriate spatial regularity of elements of the function space $\mathcal{X}$, one seeks to discretize, inevitably reducing a distributed pointwise constraint to some finite dimensional inequalities applied to the discretized approximation. We do not explore this here, but note the relevance of the present questions to the justification of some computational approaches of this nature.

Remark 4.5. Suppose the functions $\varphi \in \Phi$ need not be scalar-valued and we instead would be considering an apparent generalization to the intersection $\mathcal{A}=\bigcap_{\varphi \in \Phi} \mathcal{A}_{\varphi}$ where

$$
\varphi: \mathcal{X} \rightarrow \mathcal{Z}^{\varphi} \quad \mathcal{A}_{\varphi}=\left\{x \in \mathcal{X}: \varphi(x) \in \mathcal{C}^{\varphi} \subset \mathcal{Z}^{\varphi}\right\}
$$

with each $\mathcal{C}^{\varphi}$ a closed convex cone in $\mathcal{Z}^{\varphi}$; without loss of generality we take the vertex of each $\mathcal{C}^{\varphi}$ to be $0 \in \mathcal{Z}^{\varphi}$. We assume each such $\varphi \in \Phi$ is Fréchet differentiable near $\bar{x} \in \mathcal{A} \subset \mathcal{X}$ and, generalizing (12), we set

$$
\omega^{f}=\varphi(\bar{x}) \in \mathcal{Z}^{\varphi} \quad \xi^{\varphi}=\varphi^{\prime}(\bar{x}) \in L\left(\mathcal{X} \rightarrow \mathcal{Z}^{\varphi}\right)
$$

and assume $\xi^{\varphi}$ is surjective. Note that each $\mathcal{C}^{\varphi}$ can be written as

$$
\mathcal{C}^{\varphi}=\left\{z \in \mathcal{Z}^{\varphi}:\langle\zeta, z\rangle \geq 0 \text { for all } \zeta \in \Lambda^{\varphi}\right\}
$$

for a suitable set $\Lambda^{\varphi} \in\left[\mathcal{Z}^{\varphi}\right]^{*}$ and set

$$
\psi^{\zeta}: \mathcal{X} \rightarrow \mathbb{R}: x \mapsto\langle\zeta, \varphi(x)\rangle \text { for each } \zeta \in \Lambda^{\varphi}
$$

[Note that with $\zeta \neq 0$ we have $\left[\psi^{\zeta}\right]^{\prime}(\bar{x})=\zeta \xi^{\varphi} \neq 0$ by the assumed surjectivity of $\xi^{\varphi}$.] Then

$$
\mathcal{A}_{\varphi}=\bigcap_{\zeta \in \Lambda^{\varphi}} \mathcal{A}^{\psi^{\zeta}} \quad \mathcal{A}^{\psi}=\{x \in \mathcal{X}: \psi(x) \geq 0\}
$$

and

$$
\mathcal{A}=\bigcap_{\psi \in \Psi} \mathcal{A}^{\psi} \quad \Psi=\bigcup_{\varphi \in \Phi}\left\{\psi^{\zeta}: \zeta \in \Lambda^{\varphi}\right\}
$$

so this apparently more general situation reduces to our previous one: the principal difficulties would seem to be finding suitable formulations of the normalization of $\xi^{\varphi}$, the definition of $\Phi^{\omega}$, etc., so as to be able to state new 
hypotheses corresponding to (11), (15), (16) in terms of these $\varphi, \mathcal{C}^{\varphi}, \cdots$.

Of course there would be no such reduction if the cones $\mathcal{C}^{\varphi}$ would not be convex, but we are hopeful in conjecturing that the approach of this paper might be useful in obtaining a suitably corresponding result when each $\mathcal{C}^{\varphi}$ would be a polyhedral cone in a finite dimensional space with a bound on the dimensions and the number of faces involved in this presentation.

\section{References}

[1] J.M. Borwein and Q.J. Zhu, Techniques of Variational Analysis, SpringerVerlag, New York, 2005.

[2] K.A. Hoffman and T.I. Seidman, A Variational Characterization of a Hyperelastic Rod with an Impenetrable Tube, in preparation.

[3] B.S. Mordukhovich, Variational Analysis and Generalized Differentiation, I: Basic Theory, Springer-Verlag, Berlin, 2006.

[4] B.S. Mordukhovich, Variational Analysis and Generalized Differentiation, II: Applications, Springer-Verlag, Berlin, 2006. 